\title{
Expert Discussion: Highlights from the San Antonio Breast Cancer Symposium, San Antonio, December 8-11, 2020
}

\author{
Volkmar Müller $^{\mathrm{a}}$ Veronique Dieras $^{\mathrm{b}}$ Fatima Cardoso $^{\mathrm{c}}$ David Cameron $^{\mathrm{d}}$ \\ Javier Cortes ${ }^{e}$ \\ aDepartment of Gynecology, University Medical Center Hamburg-Eppendorf, Hamburg, Germany; \\ ${ }^{b}$ Breast Oncology, Eugène Marquis Centre, Rennes, France; ' $\mathrm{C}$ Breast Unit, Champalimaud Clinical Center, \\ Lisbon, Portugal; ${ }^{d}$ Cancer Research UK Edinburgh Centre, MRC Institute of Genetics and Molecular Medicine, \\ Western General Hospital, Edinburgh, UK; ${ }^{\mathrm{I}} \mathrm{OOB}$ Institute of Breast Cancer, Quiron Group, Barcelona, Spain
}

\section{Müller: Which results presented at the 2020 \\ San Antonio Breast Cancer Symposium would you consider to be the most likely to be practice-changing?}

Dieras: Low-risk gene expression prognostic classifiers and chemotherapy benefit were assessed in 2 large-scale trials of patients with node-negative tumors, i.e., TAILOR-X (oncotype Dx) and MINDACT (MammaPrint). The RxPONDER study evaluated the impact of chemotherapy added to endocrine therapy in $>5,000$ patients with luminal tumor and node-positive (1-3+) and a recurrence score of $\leq 25$ (Kalinsky K. et al.). In this interim analysis, the 21-gene RS 0-25 was prognostic, but it did not predict the benefit of chemotherapy which was similar across RS 0-25. Postmenopausal women with RS 0-25 did not benefit from adjuvant chemotherapy in any subgroup whereas premenopausal women with RS 0-25 had benefit from the addition of chemotherapy to endocrine therapy $(46 \%$ decrease in invasive disease-free survival [IDFS] events; benefit was observed across premenopausal subgroups), with a $53 \%$ decrease in deaths, leading to a 5 -year OS absolute improvement of 1.3\%.

Cameron: The only hint that RS score is predictive for chemotherapy efficacy was in the premenopausal group where the benefit was greater in those with a higher score;
RS subgroups 0-13: 3.9\% (25 events) and 14-25: 6.2\% (107 events). However, for me, the study confirms that RS is a prognostic tool, but there is still no hard evidence that it is predictive of chemotherapy sensitivity, since the absolute gains will always be higher in those with higher RS scores because their risk of recurrence is higher. What we need is evidence that the relative (not absolute) benefit of chemotherapy increases along with RS score. This study was designed to demonstrate this, but it failed to do so.

In fact, RxPONDER replicated the results already observed in TAILOR-X, i.e., that premenopausal patients with an intermediate RS (16-25) may derive chemotherapy benefit. The hypothesis was that ovarian function suppression effect explains the chemotherapy benefit in younger women with a low-risk signature. Today, we cannot definitively confirm this hypothesis, and more data are needed. A longer follow-up will add more data on the menopausal status after chemotherapy in young women.

Cardoso: There was also the presentation of the ADAPT trial, that used a different approach, namely a short preoperative standard endocrine therapy (tamoxifen or aromatase inhibitor) for patients who would usually be candidates for adjuvant chemotherapy according to conventional prognostic criteria. Based on the biological response (assessed by Ki-67) and RS, systemic adjuvant therapy was determined. 
The MINDACT trial presented results in the group of patients considered to be at a low clinical risk. In this group, MammaPrint retained its prognostic value, but for patients with a high genomic risk and a low clinical risk, chemotherapy did not provide a significant benefit.

Some important messages that can be taken from all available studies using genomic signatures are: (1) these signatures are prognostic but not predictive biomarkers, (2) identification of worse prognosis is not always equivalent to a better response to chemotherapy; in fact, for this breast cancer (BC) subtype, optimization of endocrinebased therapy is most probably a better option than chemotherapy (either through ovarian function suppression and/or the use of CDK4/6 inhibitors), and (3) genomic tests should always be used in combination with clinical and pathological factors, and not in isolation.

\section{Cameron: I agree!}

Dieras: I would like to add PRIME2, but I feel conflicted. It showed that the omission of postoperative radiotherapy had no detrimental effect on distant recurrence or overall survival (OS) of older women with luminal BC given adjuvant hormonal therapy after breast-conserving surgery. There was the expected reduction in local relapse, but no effect at all on OS. This is, in part, because these older women have significant competing risks of mortality, including cardiovascular deaths that might be increased by postoperative radiotherapy. To me, this means an older woman with endocrine-sensitive lower-risk disease can keep her breast and avoid radiotherapy if she wants, knowing that her survival will not be compromised. This might be attractive for those with mobility problems and those who live a long way from radiotherapy sites.

Cortes: We have had important clinical study results from the SABCS this year. The most important data with implications for clinical practice came from RxPONDER. Based on retrospective data, postmenopausal patients with hormone-receptor-positive/HER2-negative BC, even with positive nodes (usually 1 or 2), and an oncotype DX RS $<25$, were considered to be treated with endocrine therapy alone. Now, the prospective data (RxPONDER study) showed a 5-year IDFS of 91.9\% with endocrine therapy alone, and 91.6 if chemotherapy was also administered. On the other hand, and aligned with TAILORx, in premenopausal patients, we need better biomarkers to define who will not need chemotherapy.

A second very important study is PENELOPE-B. Based on the monarchE study, patients with high-risk ER+/ HER2- BC might benefit from 2 years of adjuvant abemaciclib added to endocrine therapy. However, PENELOPE-B showed that, at least with palbociclib, this might not be true. We need to clarify if this was due to the dif- ferent patient population, the different toxicities, longer follow-up, or the different drugs/doses before using CDK4/6 inhibitors widely in this setting in clinical practice.

\section{Müller: Did you find developments in targeted therapies for breast cancer patients that will make their way into clinical practice?}

Dieras: After the release at ESMO of the PALLAS and monarchE data that evaluated the addition of a CDK4/6 inhibitor to endocrine therapy in the adjuvant setting, the PENELOPE-B data were eagerly awaited. In this trial, patients treated with neoadjuvant chemotherapy for $\mathrm{HR}+/ \mathrm{HER} 2-\mathrm{BC}$ and no pathological complete response at surgery were randomized to receive palbociclib/placebo for 1 year in addition to endocrine therapy. The trial with the longest median follow-up of 43 months (PENELOPE-B didn't meet its primary end point for IDFS. The monarchE data were updated, with a slight increase in median follow-up of 19.1 months; abemaciclib combined with standard endocrine therapy continued to demonstrate a reduction in the risk of developing IDFS and DFRS events for patients with high-risk $\mathrm{HR}+1$ HER 2 - early BC. Important to note, only $25 \%$ of patients completed the 2 -year treatment. The 3 trials of CDK $4 / 6$ inhibitor in the early setting are different in their population, duration of CDK4/6 inhibitor treatment, follow-up, and discontinuation rates. It is really important to have a longer follow-up for the monarchE study to assess the rate of discontinuation of all patients as well as the true impact on the incidence of late relapse in this high-risk population.

For immune checkpoint inhibitors, after the negative results of IMpassion131 (paclitaxel $+/$ - atezolizumab) presented at ESMO, it was very important to have updates of KEYNOTE 355: chemotherapy plus or less pembrolizumab as first-line therapy in metastatic triple-negative BC (mTNBC) (Rugo H. et al.). Pembrolizumab plus chemotherapy resulted in a statistically significant improvement in progression-free survival (PFS) versus chemotherapy alone for the first-line treatment of PD-L1-positive (CPS $\geq 10)$ mTNBC. Important to note, in the subgroup analysis, PFS was improved regardless of the chemotherapy partner (notably, paclitaxel). But with these 3 randomized trials ( 2 positive and 1 negative), the optimal chemotherapy partner has still not been defined. Clearly, the degree of positivity of PD-L1 is associated with the efficacy of immune-oncology therapeutics (IO), but the companion tests are different across studies, and the threshold has to be better defined. As OS is still dismal, the impact of IO in TNBC will likely be more relevant in the early setting in which the addition of check- 
point inhibitor clearly increases the pathological complete response rate, considered a good surrogate marker. However, in the early setting, the challenge will be to clearly identify the patients who need the addition of IO in order not to overtreat.

Despite the results of the phase II LOTUS trial, the addition of ipatasertib to paclitaxel for PIK3CA/AKT1/ PTEN-altered advanced TNBC in the IPATunity130 cohort A did not significantly improve PFS (Dent R. et al.).

Cardoso: With the currently available data, the use of CDK4/6 inhibitors for early BC should be carefully balanced with increased short and long-term toxicity, as well as with compliance issues. With 2 negative trials (PALLAS and PENELOPE-B), there is no current role for palbociclib in this setting; the role of abemaciclib, despite encouraging early results, should wait for longer followup and mature results. It is concerning to see that, in PENELOPE-B, there was an initial separation of the KM curves similar to what is seen in monarchE, and that, with longer follow-up, that difference disappeared. Thus, because the majority of relapses in luminal $\mathrm{BC}$ occur $>5$ years after diagnosis, it will only be with longer follow-up that we can be sure of the sustained benefit from adjuvant CDK4/6 inhibitor, if any exists.

In the metastatic setting, 2 disappointing negative trials were presented: the IPATunity130 trial evaluating ipatasertib to paclitaxel for PIK3CA/AKT1/PTEN-altered advanced TNBC and the E2112 trial evaluating entinostat, an HDAC inhibitor, for ER+/HER2- advanced BC. Both trials were started after good results in phase 2 trials, especially entinostat which had shown very interesting OS benefit in the ENCORE phase 2 trial. These results clearly call again for caution about overinterpreting results from early development trials, which often are not confirmed in randomized phase 3 trials. Targeting these 2 important pathways should continue to be explored, and a deep analysis for the causes of negative results, including translational research, should be undertaken to help future trials.

Cortes: In addition to PENELOPE-B, I would like to highlight 3 studies: KN355, IPATunity, and DESTINYBreast 01 . Although the first coprimary end point of KN355 (chemotherapy with either placebo or pembrolizumab) was presented at ASCO 2020, important data were also presented at the SABCS. As expected, responses were also higher with pembrolizumab-based treatment. Of great interest, the combinations of paclitaxel or nab-paclitaxel and pembrolizumab seem to offer similar efficacy data when compared with paclitaxel or nab-paclitaxel single agents, respectively. It is important to note the low numbers in this subanalysis, but the same effect was observed in all subgroups (PDL CPS $\geq 10$, PDL CPS $\geq 1$, and in the intention- to-treat population). This observation is of particular interest after obtaining the IMpassion 131 data. In this study, presented at ESMO 220, paclitaxel and atezolizumab treatment was not superior to paclitaxel and placebo. We were disappointed with the IPATunity130 study. Based on previous AKT inhibitor clinical trials (LOTUS and PAKT) showing interesting data in patients with a PIK3CA/ AKT1/PTEN-altered pathway, we didn't expect such clear negative results. The combination of ipatasertib and paclitaxel did not translate into an improvement in PFS in this patient population. Some questions include: Are there clinical differences between ipatasertib and capivasertib? Might AKT inhibitors work with other agents? Is the firstline setting the optimal scenario to test these agents? What is very clear to me is the interest about these compounds. More clinical data are needed to address the role of this pathway in patients with TNBC. Finally, Shanu Modi updated the results of DESTINY-Breast01, a large-scale phase 2 study with DS8201 in heavily pretreated HER2+ metastatic BC. An impressive objective response rate (ORR), PFS, and OS of $61.4 \%, 19.4$ months, and 24.6 months, respectively, were reported.

\section{Müller: What are important breast cancer trials currently recruiting that were presented in the "Trials in Progress Session"?}

Cortes: I would like to mention the GLORIA study. After the CREATE-X study, showing an improvement in DFS and OS with adjuvant capecitabine in patients with a nonpathologic complete response (CR) after neoadjuvant treatment, the GLORIA study was a randomized phase 3 study of the anti-Globo $\mathrm{H}$ vaccine, adagloxad simolenin (OBI-822)/OBI-821, in patients with high-risk TNBC, defined as a $\geq 1 \mathrm{~cm}$ residual primary or $\geq 1 \mathrm{~cm}$ residual axillary node after neoadjuvant treatment or pathological stage IIB or III disease treated with adjuvant chemotherapy. Globo $\mathrm{H}$ is a glycan isolated from the $\mathrm{BC}$ cell line MCF-f that is overexpressed on a variety of epithelial cell tumors. Globo H seems to promote immunosuppression, tumor survival, and angiogenesis. The primary end point was IDFS.

\section{Müller: Which further aspects did you find to be of relevance?}

Dieras: The design of the ADAPT trial is very attractive with the assessment of the effect of a short endocrine therapy exposure on Ki-67 with the aim of identifying patients whose tumors are clearly endocrine-sensitive and who may thus be spared undergoing chemotherapy. The patients with RS $<12$ and those with RS 12-25 with Ki-67 
$<10 \%$ at week 3 do equally well, including patients with 1-2 positive nodes. An algorithm for predicting endocrine response is proposed but feasibility needs to be confirmed in larger cohort.

The CONTESSA trial evaluated a new oral taxane, tesetaxel, in combination with capecitabine, versus capecitabine monotherapy in metastatic $\mathrm{BC}$ patients who had received a taxane in the adjuvant setting.

Cardoso: The correlative biomarker analysis in the MONALEESA trials, that looked into intrinsic subtypes and the efficacy of ribociclib, yielded very interesting results, namely a substantial effect of CDK4/6 inhibition of the HER2-enriched subtype, no efficacy in the basal subtype, and the potential role of subtyping to identify luminal tumors that can be adequately treated with endocrine therapy alone (i.e., luminal A).

The CONTESSA trial should be the last of this type of trials for metastatic BC. Combination chemotherapy is rarely necessary and evaluation of PFS in a trial comparing a single agent versus a combination is a useless end point. A much more appropriate trial design would have been a head-to-head comparison with capecitabine alone or with i.v. taxane alone. The time has come for regulators and investigators to agree on a faster and more appropriate development pathway for drugs that might not bring huge benefits in efficacy but can have a positive impact on quality of life, toxicity, and/or accomodate the patient (i.e., oral or s.c. formulations). The same way that biosimilars are developed through a different and faster methodology, so new formulations should be.

Cortes: In addition to CONTESSA, which explored the combination of the oral taxane tesetaxel with capecitabine in the first-line setting in HR+/HER2- MBS patients, I would like to mention the biomarker analysis of the ASCENT trial. This study evaluated the role of sacituzumab govitecan in patients with pretreated TNBC. It showed an improvement in ORR, PFS, and OS compared with the treatment of the physician's choice (TPC). Although subgroup analyses are always exploratory in nature because they are not powered enough, the study revealed that the clinical benefit of sacituzumab over TPC was irrespective of Trop-2 expression or germline BRCA1/2 status.

\section{Conflict of Interest Statement}

V.M. received: speaker honoraria from Amgen, Astra Zeneca, Daiichi-Sankyo, Eisai, Pfizer, MSD, Novartis, Roche, Teva, and Seattle Genetics; consultancy honoraria from Genomic Health, Hexal, Roche, Pierre Fabre, Amgen, ClinSol, Novartis, MSD, Daiichi-Sankyo, Eisai, Lilly, Tesaro, Seattle Genetics, and Nektar; institutional research support from Novartis, Roche, Seattle Genet- ics, and Genentech; and travel grants from Roche, Pfizer, and Daiichi Sankyo.

V.D.: stock options: none; travel expenses: Roche, Novartis, Pfizer, Lilly, AstraZeneca, and Daiichi Sankyo; honoraria: Consultant/ Advisory Boards of Roche/Genentech, Novartis, Lilly, Pfizer, AstraZeneca, Eisai, AbbVie, MSD, Daiichi Sankyo, Seattle Genetics, and Pierre Fabre Oncologie; speaker: Roche, Novartis, Pfizer, Lilly, Astra Zeneca, Daiichi Sankyo, and Seattle Genetics.

F.C.: personal financial interest in the form of a consultancy role for Amgen, Astellas/Medivation, AstraZeneca, Celgene, Daiichi-Sankyo, Eisai, GE Oncology, Genentech, GlaxoSmithKline, Macrogenics, Medscape, Merck-Sharp, Merus BV, Mylan, Mundipharma, Novartis, Pfizer, Perre-Fabre, priME Oncology, Roche, Samsung Bioepis, Sanofi, Seagen, and Teva; institutional financial support for clinical trials from Amgen, AstraZeneca, BoehringerIngelheim, Bristol-Myers-Squibb, Daiichi, Eisai, Fesenius GmbH, Genentech, GlaxoSmithKline, Ipsen, Incyte, Nektar Therapeutics, Nerviano, Novartis, Macrogenics, Medigene, Medimmune, Tigris, Varian, Wilex, and Wyeth; non-financial disclosures: Chir $\mathrm{ABC}$ Global Alliance and $\mathrm{ABC}$ Consensus Conference and Guidelines, Member/Committee Member of ESMO, ESO, EORTCBCG, IBCSG, SOLTI, ASCO, AACR, EACR, SIS, and ASPIC.

D.C: Non-personal specific and non-specific: Pfizer Limited, Celldex Therapeutics Inc., Astrazeneca, Celgene Corp., Samsung Bioepis Co. Ltd. (Korea), ELI LILLY \& Co., Roche Products Ltd., Novartis Pharma AG, Merck Sharp Dohme Ltd., PUMA Biotechnology, Inc., Clovis Oncology, Daiichi Sankyo, USA, Eisai, Exact Therapeutics, G1 Therapeutics, GSK (Glaxo SmithKline), Seattle Genetics, and Bexon/Zymeworks Biopharmaceuticals Inc.

J.C.: consulting/advisor: Roche, Celgene, Cellestia, AstraZeneca, Biothera Pharmaceutical, Merus, Seattle Genetics, Daiichi Sankyo, Erytech, Athenex, Polyphor, Lilly, Servier, Merck Sharp \& Dohme, GSK, Leuko, Bioasis, Clovis Oncology, Boehringer Ingelheim, and Kyowa Kirin; honoraria: Roche, Novartis, Celgene, Eisai, Pfizer, Samsung Bioepis, Lilly, Merck Sharp \& Dohme, and Daiichi Sankyo; research funding to the institution: Roche, Ariad pharmaceuticals, AstraZeneca, Baxalta GMBH/Servier Affaires, Bayer Healthcare, Eisai, F. Hoffman-La Roche, Guardanth Health, Merck Sharp \& Dohme, Pfizer, Piqur Therapeutics, Puma C, and Queen Mary University of London; stock, patents, and intellectual property: MedSIR; travel, accommodation, and expenses: Roche, Novartis, Eisai, Pfizer, and Daiichi Sankyo.

\section{Participants}

Prof. Dr. med. Volkmar Müller

Konservative gynäkologische Onkologie

Klinik und Poliklinik für Gynäkologie,

Universitätsklinikum Hamburg-Eppendorf

Martinistrasse 52, DE-20246 Hamburg (Germany)

vmueller@uke.uni-hamburg.de

Dr. Véronique Dieras

Centre Eugène-Marquis

Avenue Bataille Flandres-Dunkerque

FR-35042 Rennes (France)

v.dieras@rennes.unicancer.fr

\section{Dr. Fatima Cardoso}

Director Breast Unit, Champalimaud Clinical Center

Av. De Brasília s/n,

PT-1400-038 Lisbon (Portugal)

fatimacardoso@fundacaochampalimaud.pt 
Prof. David Cameron

Cancer Research UK Edinburgh Centre

MRC Institute of Genetics \& Molecular Medicine

The University of Edinburgh

Western General Hospital

Crewe Road South

Edinburgh, EH4 2XR (UK)

David.Cameron@igmm.ed.ac.uk
Dr. Javier Cortes

Head, Breast Cancer Program

IOB Institute of Oncology, Madrid \& Barcelona Plaça d'Alfonso Comín, 5

ES-08023 Barcelona (Spain)

jacortes@vhio.net 